\title{
Super-Eddington Accretion in the Formation of Low-Mass X-ray Binaries and Millisecond Pulsars
}

\author{
R. F. Webbink and V. Kalogera \\ Dept. of Astronomy, Univ. of Illinois, 1002 W. Green St., Urbana, IL \\ 61801, U.S.A.
}

\begin{abstract}
Considerations of donor star stability, age, and mass transfer rate show that low-mass X-ray binaries and binary millisecond pulsars with orbital periods longer than a few days must have survived an initial phase of super-Eddington mass transfer. We review the physical arguments leading to this conclusion, and examine its implications for the apparent discrepancy between the death rate for low-mass X-ray binaries and the birth rate of binary millisecond pulsars.
\end{abstract}

\section{Introduction}

A wide variety of evolutionary scenarios have been proposed for low-mass X-ray binary (LMXB) formation (see Iben and Tutukov 1995 for some of the possibilities; Kalogera 1996) and, by extension, for binary millisecond pulsars (mPSRs). Notwithstanding the pathway leading to the LMXB state, however, age and stability considerations severely limit the range of interacting normal star-neutron star pairs which will transfer mass at sub-Eddington rates (Kalogera \& Webbink 1996), as is the norm among LMXBs. Provided that binaries survive (or possibly are even stabilized by) super-Eddington mass transfer, mass transfer rates tend to subside with time; but examples may be found among LMXBs, and certainly among binary mPSRs, of systems which could not have reached their current evolutionary state without having survived super-Eddington mass transfer.

\section{Structural and Stability Constraints}

The mass transfer rate at the onset of the LMXB phase is dictated by the stability (or lack thereof) of the donor star. For present purposes, we assume an initial gravitational mass of $1.4 \mathrm{M}_{\odot}$ for the compact, accreting star. Then, donor stars between the zero-age main sequence and the base of the giant branch become unstable to thermal time scale mass transfer if their masses exceed $\sim 1.6 \mathrm{M}_{\odot} ;$ more massive donors transfer mass at super-Eddington rates. Beyond the base of the giant branch, the more stringent constraint becomes one of dynamical stability, which restricts viable donors to $<1.08 \mathrm{M}_{\odot}$ (if total mass and orbital angular momentum are conserved). Less massive donor stars are stable against both thermal and dynamical mass transfer, and transfer mass on a nuclear time scale (accelerated perhaps at short orbital periods by angular 
momentum losses in a magnetic stellar wind). This rate depends strongly on the degenerate core mass of a giant branch donor, and itself exceeds the Eddington limit if the core mass is large, and the donor therefore rapidly-evolving. The limiting orbital period below which the mass transfer rate is sub-Eddington runs from $1^{\mathrm{d}}$ at $1.04 \mathrm{M}_{\odot}$ (the base of its giant branch) up to $\sim 220^{\mathrm{d}}$ at $0.33 \mathrm{M}_{\odot}$ (the tip of its giant branch). Stars of mass $<1.04 \mathrm{M}_{\odot}$ and solar composition take $>10^{10} \mathrm{yr}$ to reach the base of the giant branch.

\section{Conclusions}

Binary mPSRs with orbital periods exceeding $\sim 200^{\mathrm{d}}$ (very slightly longer for more massive neutron stars), such as B1718-19 and B0820+02, evidently reached that state after prolonged super-Eddington mass transfer, and may never have appeared as LMXBs if the accretion energy was degraded in a dense wind outflow. More generally, however, binary mPSRs and LMXBs with giant branch donors can have avoided super-Eddington mass transfer in reaching their present states only if they began mass transfer before the donors reached the base of the giant branch. Such systems reach terminal orbital periods of $\sim 7^{\mathrm{d}}$ (if mass transfer is conservative), implying that mPSRs and LMXBs with longer orbital periods than this ( $\sim 40 \%$ of mPSRs with nearly circular orbits) must have survived at least some super-Eddington mass transfer.

It is evident that a physical understanding of super-Eddington accretion and outflows is crucial to understanding the evolutionary relationship between LMXBs and mPSRs. This is a problem which remains to be solved theoretically, but if we take SS 433 as the paradigm for the process, then similar objects should occur with birth rates at least comparable to those of LMXBs and mPSRs (albeit with much shorter lifetimes).

Acknowledgments. This research was supported by National Science Foundation grant AST92-18074.

\section{References}

Iben, I. Jr., \& Tutukov, A. V. 1995, ApJS, 100, 233

Kalogera, V. 1996, ApJ, in press

Kalogera, V., \& Webbink, R. F. 1996, ApJ, 458, 301 